\title{
A comprehensive and robust procedure for obtaining the nofit polygon using Minkowski sums
}

\author{
JULIA A BENNELL \\ School of Management, University of Southampton, Southampton SO17 1BJ, United \\ Kingdom, j.bennell@soton.ac.uk \\ XIANG SONG \\ School of Management, University of Southampton, Southampton SO17 1BJ, United \\ Kingdom, x.song@soton.ac.uk
}

The nofit polygon an important tool in the area of irregular shape stock cutting problems. It provides efficient handling of the geometric characteristics of the problem. The paper presents a new algorithmic procedure for deriving this tool.

Submitted: June 2005

Subject classification: cutting stock, geometric, algorithm Area of review: Optimisation 


\begin{abstract}
The nofit polygon is a powerful and effective tool for handling the geometric requirements of solution approaches to irregular cutting and packing problems. Although the concept was first described in 1966, it was not until the early 90s that the general trend of research moved away from direct trigonometry to favour the nofit polygon. Since then, the ability to calculate the nofit polygon has practically become a pre-requisite for researching irregular packing problems. However, realisation of this concept in the form of a robust algorithm is a highly challenging task with few instructive approaches published. In this paper, a procedure using the mathematical concept of Minkowski sums for the calculation of the nofit polygon is presented. The described procedure is more robust than other approaches using Minkowski Sum knowledge and includes details of the removal of internal edges to find holes, slits and lock and key positions. The procedure is tested on benchmark data sets and gives examples of complicated cases. In addition the paper includes a description of how the procedure is modified in order to realise the inner-fit polygon.
\end{abstract}




\section{INTRODUCTION}

The paper specifically addresses the geometric calculations required for tackling cutting and packing problems involving irregular shapes. Such problems are common in manufacturing processes and occur whenever a piece of irregular shape is to be cut from a sheet of stock material. Examples include dye-cutting in the engineering sector, parts nesting for shipbuilding, marker layout in the garment industry, and leather cutting for shoes, furniture and other goods. Here we consider that shapes are irregular if they are; polygonal, i.e no arcs; simple, i.e. do not self-cross; and non-rectangular. Even when all the components are rectangular the problem of finding layouts that minimize waste is known to be NP-hard. Where irregular components are involved an extra dimension of complexity is generated by the geometry.

The precise requirements of a good layout will differ from industry to industry and this has lead to a variety of algorithmic approaches. In spite of their differences, all the methods have a common requirement in which they need to be able to identify whether a layout is feasible or not, i.e. do any of the pieces overlap. Early research handled this problem in a number of ways. Adamowicz and Albano (1976) chose to nest pieces into simpler shapes where the geometry can be more easily calculated. If the shapes are used directly then the intersection of pieces can be handled by direct trigonometric approaches such as the D function (Mehadavan, 1984; Konopasek, 1981). Alternatively the stock sheet and the pieces can be approximated as grid squares, often referred to as the raster method. Hence, if a piece occupies, fully or partially, a grid square it is coded as occupied (Oliveira and Ferreira, 1993; Babu and Babu, 2001). 
Although all these approaches have merit, it is widely recognized that the nofit polygon (NFP) is more efficient, provided you have a robust and efficient NFP generator, and has become the principle approach for handling the geometry in nesting problems. Unfortunately, some researchers believe that despite the value of this tool, its introduction may have stifled research into this variant of packing problems. Wäscher, Haußner and Schumann (2005) reports that there have been only 21 publications in irregular problems in the last 10 years. Researchers attribute this to the fact that the realization of the NFP as a robust algorithm is, in itself, a highly challenging task. Those considering embarking on research into irregular shaped packing may be discouraged by the significant investment of time required in first developing an NFP generator. Hence, it is essential that robust and easily realizable algorithms are available in order to facilitate new interest into this important problem.

The primary purpose of this paper is to introduce a new procedure for calculating the NFP. The method is developed from the theory of Minkowski sums and builds on the principles proposed by Ghosh (1993) and by Bennell, Dowsland and Dowsland (2001). Further, the paper includes an algorithmic procedure for eliciting the true boundary of the NFP, including holes, slits and exact fits. The next section outlines the most commonly cited approaches for calculating the NFP and points out their positive features and disadvantages. Section 3 reviews in more detail the Minkowski sum approach. This is followed by a description of our new procedure based on Minkowski sums. Section 5, develops our approach for removing redundant internal points and therefore identifying the true boundary. In both cases the full algorithmic steps are provided. Section 6 outlines 
the modification required in order to determine the inner-fit polygon. Finally, we develop some theoretical and empirical analysis of the approach to demonstrate its robustness.

\section{DOCUMENTED APPROACHES FOR GENERATING THE NOFIT POLYGON}

The nofit polygon (NFP) is a combination of the properties of two component polygons that, as a result, represents all the relative positions of the two polygons in which they either touch or overlap. It is well documented that the NFP can reduce the complexity of detecting overlap between two pieces from $O(n m+n+m)$, where $n$ and $m$ are the number of edges in each polygon, obtained from direct trigonometry, to a simple point inclusion test of $O(k)$, where $k$ is the number of edges in the NFP. Full explanations of the concept can be found in (Mehadevan, 1984; Ghosh, 1991; Bennell, 1998; Bennell Dowsland and Dowsland, 2001), where the most intuitive description is found in Cunningham-Green (1989), who describes the motion of one polygon sliding around the boundary of the other; often referred to as the orbiting method. Figure $1 \mathrm{a}$ and $1 \mathrm{~b}$ illustrates the motion of polygon $B$, the orbiting polygon, sliding around $A$, the fixed polygon, tracing the locus of a reference point on $B$. He also notes that when both polygons are convex, the NFP is an exact replication of the edges of both polygons, with opposite orientation, sorted into their slope order. Figure 1c shows the edges of both polygons, where $A$ has counterclockwise orientation and $B$ has clockwise orientation, sorted into slope order; these can be directly mapped onto the NFP in figure $1 \mathrm{~b}$. Note that this role and orientation of polygons $A$ and $B$ will be adopted for the remainder of the paper. Cunningham-Green's (1989) observations underpin two of the most common 
approaches to generating the NFP; the orbiting method that simulates the sliding motion, and Minkowski sums that sort the edges according to the the slope order and edge precedence, i.e the sequential order of edges around the polygons. A further approach commonly employed is that of decomposition. A brief description of each is provided here.

(a)

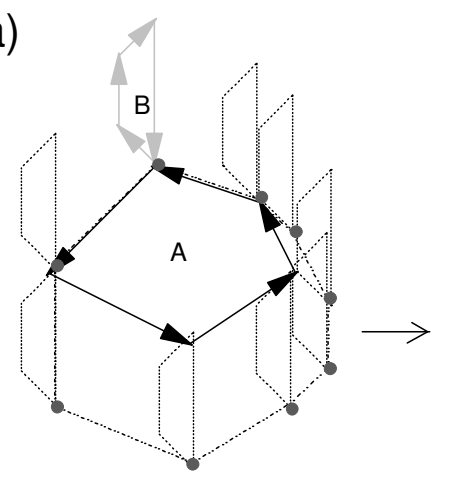

- Reference point on B

(c)

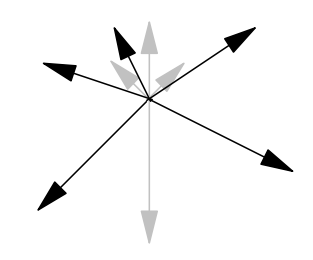

(b)

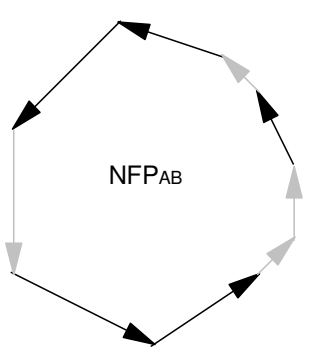

Figure 1: The locus of the reference point on $B$ traces the NFP as it slides around $A$.

This is equivalent to connecting the edges in slope order.

\section{Minkowski sum}

Clearly, when both component polygons are convex the NFP is very simple to calculate by sorting the edges into slope order. Further, when one of the polygons is 
convex and the other is an arbitrary simple polygon, the NFP can still be easily obtained from the slope order and the precedence of the edges. In this case, the NFP is obtained by forming an edge list that follows the precedence of the simple polygon, assigned as polygon $A$, in a counterclockwise direction, and adding the edges of the convex polygon, assigned polygon $B$, to the list whenever they are encountered in the slope order. Due to the concavities in $A$, the precedence will necessitate a clockwise turn through the slope order, if the edges of the convex polygon are encountered in the clockwise direction; they are included in the edge list with negative direction. Note that this also retains the precedence of the edges of the convex polygon. Unfortunately the resulting polygon created from this edge list is complex and further computation is required to remove edges or parts of edges that are not part of the boundary of the NFP. Figure 2 illustrates the tracking of the precedence order of a simple polygon through the slope order.

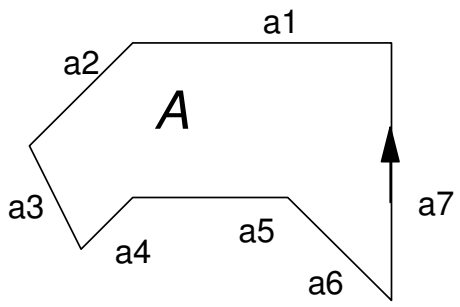

polygon

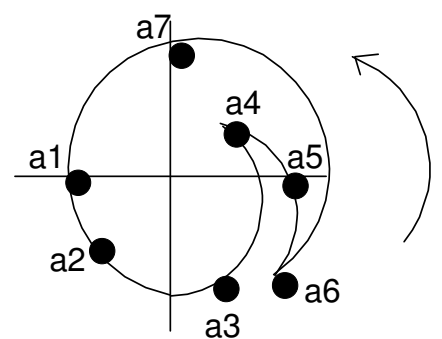

slope diagram

Figure 2: A simple polygon and respective slope order

It is worth noting that even in the convex-simple case, the NFP may contain holes. These represent a non-overlapping placement position within a concavity that cannot be 
encountered through sliding. Such cases will be discussed later in the paper. When both polygons contain concavities, following the precedence of both polygons, simultaneously, becomes impossible without further modification to the approach. Since it is these principles that form the basis of the Minkowski sum approaches presented in this paper, these issues will be discussed in later sections.

\section{Orbiting method}

An alternative approach is to use the orbiting method (Mahadevan, 1984). This approach attempts to simulate the sliding motion of one polygon around the other. When both polygons are convex, this is equivalent to sorting the edges in slope order. However, when one or both of the polygons have concavities, the full extent of some edges may not be available to slide along without generating overlap. Mahadevan's approach calculates the nature of the touching vertices and edges, at a given point, in order to identify the next edge to slide along; this is the translation vector. He then projects forward the vertices of the orbiting polygon and projects backward the vertices of the fixed polygon in order to identify the closest point of intersection. The orbiting polygon is then translated along the translation vector to the point of the closest intersection. The key criticism of this approach is that it can only identify the external boundary of the NFP and any holes that may exist will be missed. Burke et al (2005) have proposed some modifications to Mehadevan's approach that improves the computational efficiency and permit the identification of holes. They first find the outer face of the NFP using the principles of Mehadevan's sliding approach, while recording each edge of the polygons that have been partially or fully traversed. The edges that are not flagged are then 
candidates for possible holes. A process of identifying all feasible touching start position is performed for the candidate edges. If a feasible start position is found, the sliding approach is performed again from that starting point. This continues until all edges not flagged have been investigated.

\section{Decomposition}

Given the comparative complexities of the described approaches when one or both polygons are simple, decomposing the component polygons into suitable sub-polygons is an attractive option. Examples in cutting and packing literature include convex decomposition (Watson and Tobias, 1999) and star shaped decomposition (Li and Milenkovic, 1995). As previously described, the NFP of two convex polygons is trivial. Li and Milenkovic selected star shaped polygons since the NFP of two star shaped polygons is also star shaped. Hence, in generating the sub-NFP, they need only be concerned with the outer boundary.

Although decomposition simplifies the core NFP operation, it also generates two further issues; efficient decomposition and robust recombining of the sub-NFPs. Agarwal Flato and Halperin (2002) investigated these issues for convex decomposition. They determined that optimal decomposition could significantly reduce the number of subNFPs required, but this benefit did not out weigh the computational cost of the decomposition process. Recombination provides further challenges, since if edges from two sub-NFPs coincide or cross in and out of each other, careful analysis must be performed to detect whether these edges are part of the boundary of the NFP. Agarwal, 
Flato and Halperin (2002) found that the recombination operation was the most computationally expensive and report relatively high computation times.

A recent development in handling the geometric properties of irregular packing problems, in both two and three dimensions, is that of the Phi-function (Stoyan et al, 2001, 2002). Although phi-functions are not strictly nofit polygons, they are a related concept and have proved to be both efficient and effective. The Phi-function is able to determine the distance between two polygons and therefore whether they overlap. Stoyan et al analytically construct phi-functions for all primary objects; rectangles, circles and other convex polygons. As a result, arbitrary polygons or parallelepipeds can be handled by representing them as a finite combination (union, intersection, complement) of primary objects.

All of the methods described have been somewhat successful. However, all experience difficulties when; the problem instance becomes complex, for example, degenerate cases where one or more dimension fits exactly into a concavity; computational times can be large; and the algorithm proposed difficult to realize. In this paper we will further develop the Minkowski sum approach and present a robust, efficient and simple algorithm. Although we do not dismiss the potential of the other approaches, a clear advantage of this approach is that the basic Minkowski sum can be obtained through simple rules designed to list the edges according to the precedence of both polygons while sorting in slope order. For all the described methods, the identification of holes and degenerate cases is somewhat laborious.

\section{APPROCHES TO FINDING THE NOFIT POLYGON USING MINKOWSKI}

\section{SUMS}


As previously described, generating the NFP of both the convex-convex and simpleconvex case can easily be solved using the slope and precedence order of the edges. Ghosh $(1991,1993)$ developed these ideas and proposed the theory of boundary addition, which can be illustrated through the use of a slope diagram. Figure 3a illustrates two polygons converted into their respective slope diagrams. Note that the polygons have opposite orientation, $A$ has counter clockwise orientation; positive, and $B$ has clockwise orientation; negative. The boundary addition theorem states that the Minkowski sum, $A \oplus-B$, which is equivalent to the NFP, can be obtained from merging the slope diagrams of $A$ and $-B$ and is given by an edge list that follows the slope order and retains the precedence of the edges of both $A$ and $-B$ through counter clockwise (positive) and clockwise (negative) turns. The simple-simple case is also comprehensively addressed by the boundary addition theorem. However, when concavities in the two polygons interact, it becomes impossible to define one path through the slope diagram that retains the precedence of both polygons. Ghosh overcame this problem by defining parallel paths, where the precedence of one or the other polygon would dominate. His approach is illustrated in figure 3. Unfortunately, when multiple concavities interact, between and within the polygons, it becomes impossible to define algorithmic rules for robustly untangling the conflicting areas. 
(a)
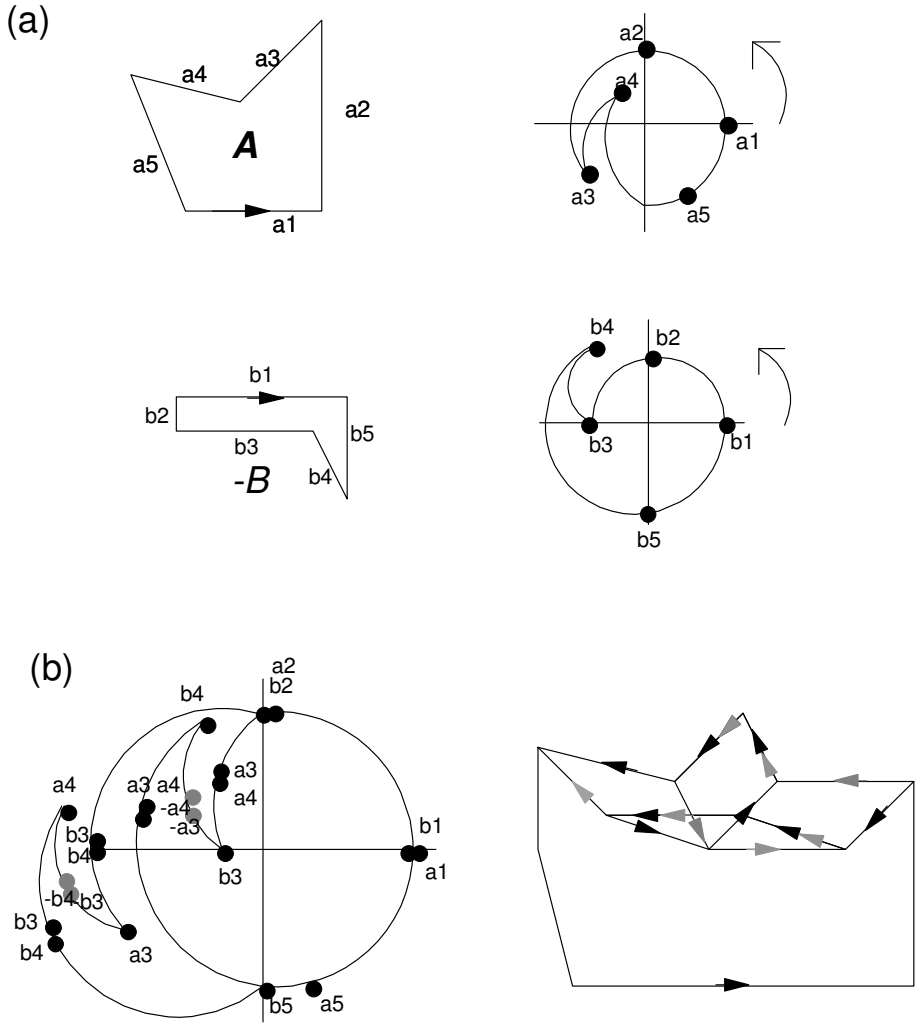

Path 1 b2, a3, a4, b3, -a4, -a3, b4, a3, a4, b5 $\} \rightarrow$ Path $2\{b 2, b 3, b 4, a 3,-b 4,-b 3, a 4, b 3, b 4, b 5\}$

Figure 3 Ghosh (1991) approach to two simple polygons with interacting concavities

Bennell, Dowsland and Dowsland (2001) propose an alternative approach to the simple-simple case. Their approach exploits the knowledge that the simple-convex case is trivial and that the Minkowski sum of a simple polygon A with the convex hull of polygon $\mathrm{B}, \mathrm{Mink}_{\mathrm{Aconv}(\mathrm{B})}$, will contain all the boundary and internal points of the original simple-simple case, $\operatorname{Mink}_{\mathrm{AB}}$. In order to generate $\operatorname{conv}(\mathrm{B})$, dummy edges are introduced that replace the edges that make up the concavities of B. Clearly these dummy edges 
appear, both positively and negatively, in $\operatorname{Mink}_{\mathrm{Aconv}(\mathrm{B}) \text {. Hence replacing the dummy edges }}$ in the edge list of $\operatorname{Mink}_{\mathrm{Aconv}(\mathrm{B})}$ by the real edges of $\mathrm{B}$, following the precedence of the edges and including $A$ edges when they are passed in the slope order, will result in $\operatorname{Mink}_{\mathrm{AB}}$.

While Bennell, Dowsland and Dowsland's approach works well on the benchmark data sets (ESICUP), further investigation highlights some ambiguity in the procedure for replacing dummy edges. This is illustrated through the example in figure 4.

Figure 4(a) illustrates the generation of $\operatorname{Mink}_{\mathrm{Aconv}(\mathrm{B})}$. It is clear that dummy edge $b d_{l}$ will slide across the vertex between edge $a_{9}$ and edge $a_{1}$. Hence appearing on the slope diagram on that traversal alone. However, we can observe in figure 4(b) that vertex $\left(a_{9}, a_{1}\right)$ can not slide along the full extent of edge $b_{2}$ due to a collision between edge $b_{3}$ and vertex $\left(a_{6}, a_{7}\right)$. However, if when replacing the dummy edge in the slope diagram, only the $A$ edges on the same traversal are considered, this collision will not be included in the boundary of the NFP. Figure 4(b) illustrates, the resulting Mink $\mathrm{AB}_{\mathrm{BB}}$ when only the current traversal is considered, and the true NFP. The problem can be resolved by including the additional edges. However, defining rules to determine the instances in which extra $A$ edges should be included has proved difficult. 

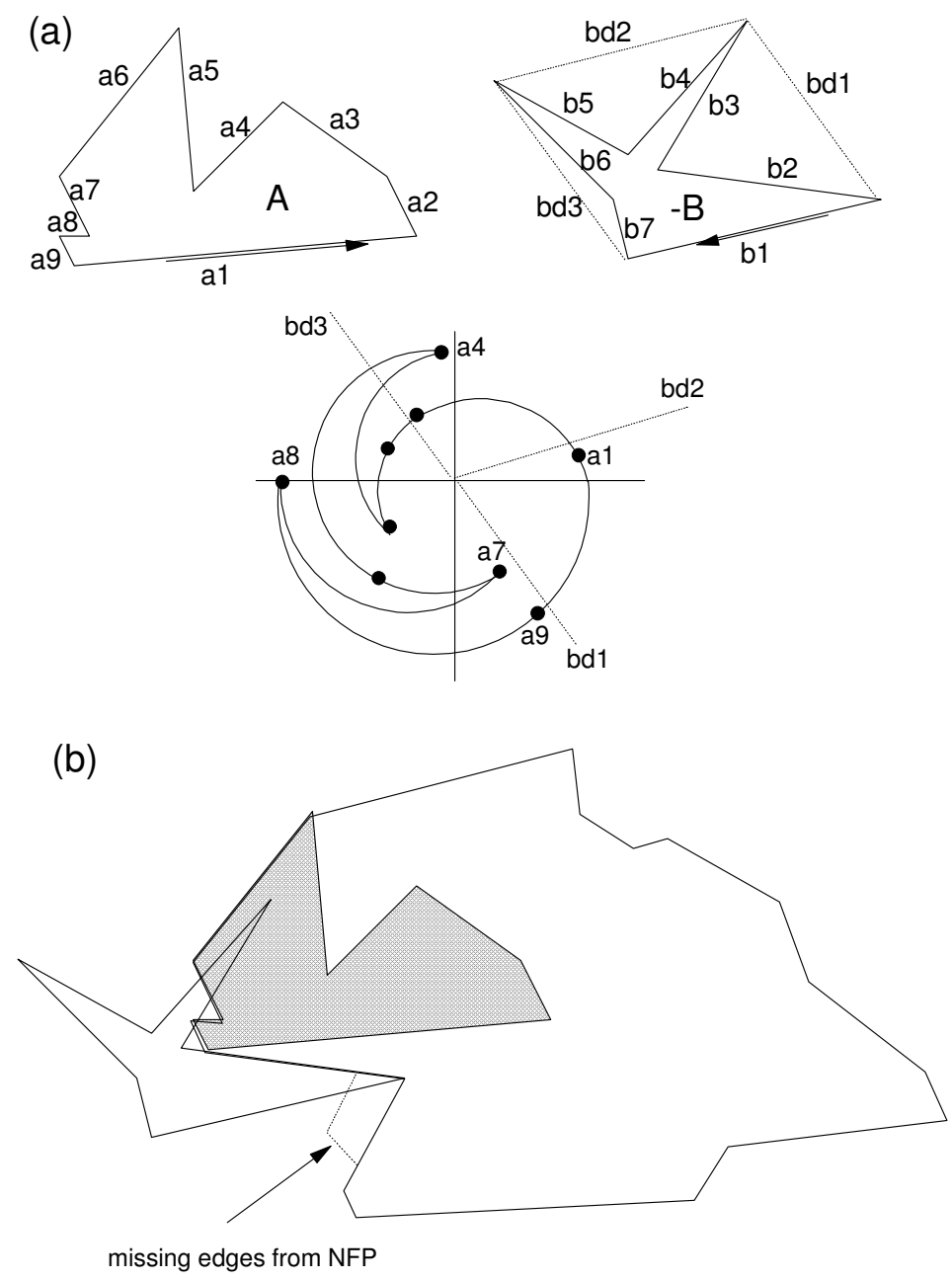

Figure 4 An example when edges may be missed when using Bennell, Dowsland and Dowsland (2001)

\section{A REVISED PROCEDURE FOR OBTAINING THE BOUNDARY OF THE NOFIT POLYGON}

The proposed new approach for finding the NFP is also based on the boundary addition theorem and inspired by the observation that the simple-convex case is trivial. Further, the new approach is simple, intuitive and removes ambiguity concerning which 
edges should be included in the edge list. The basic idea is to break polygon $B$ into groups that are in either continuous counter clockwise or clockwise order. Each of the groups can then be individually merged with the slope diagram of $A$ without conflict. When combining the merged lists, linking edges need to be included in order to maintain the precedence of the edges in each polygon. As with Ghosh and Bennell, Dowsland and Dowsland, the resulting edge list is a complex polygon, where the edges represent all the boundary edges and some internal points of the Minkowski sum. In order to have successfully generated the NFP, the edges that are not part of the boundary must be removed. The approach for finding the Minkowski sum will be first illustrated by an example and then the algorithmic procedure will be given. Removal of internal edges will be addressed in the next section.

Consider the example previously given in figure 3. If we follow the precedence of $A$, traversing from a2 to a3 we will encounter b4 before $\mathrm{b} 3$, yet the previous $B$ edge encountered had been b2. The equivalent conflict would occur in $A$ if we followed the precedence of $B$. However, if we break polygon $B$ at the vertex connecting $\mathrm{b} 3$ and $\mathrm{b} 4$, and consider the edges as a list, instead of a cycle, starting from b4, then we have b4, b5, b1, $\mathbf{b 2}$ and $\mathbf{b 3}$ in a continuous counter clockwise direction. As a result, all the edge points $B$ on the slope diagram are in the correct order, and b3, at this time, has no connection to b4. Having made this break, the procedure can be described as one of searching for the next $B$ edge on the list through following the precedence path of $A$. Hence, only the next $B$ edge is active, all others are dormant. Since the $B$ edges may be visited more than once, it is first necessary to perform an initial exploratory cycle of the merged slope order, following the precedence of $A$ and counting the number of times a $B$ edge is traversed. 
The approach applied to the example in figure 3 works as follows. Start from the first $B$ edge, b4, we search for b5. To find this we traverse a3 and a4. From b5 we search for b1 and traverse a5. From b1 we search for b2 and traverse a1. Finally we search for b3 traversing a2. Since b3 crosses the concavity of $A$, it will appear three times. This was established through the initial counting phase. Hence the search continues until all appearances of b3 have been found. Thus we obtain b4, a3, a4, b5, a5, b1, a1, b2, a2, b3, a3, -b3, a4, b3. Given that a polygon must be a complete cycle, we must now link the beginning and the end of the list. Hence from b3 we will look for b4. This requires a clockwise turn through the slope diagram traversing -a4 and -a3. Thus finally we obtain: b4, a3, a4, b5, a5, b1, a1, b2, a2, b3, a3, -b3, a4, b3, -a4, -a3.

In summary, the procedure to form the sequence follows the slope diagram of $A$ positively when the series of $B$ edges are in a counter clockwise direction and follows it negatively when the series of $B$ edges are in a clockwise direction. With this knowledge, we consider a more complex case.

In figure 5 , there is more than one concave point in polygon $A$ and $B$. Sorting $A$ and $B$ into slope order, merging the lists and following the precedence of $A$, we discover that all $B$ edges will be traversed three time with the exception of $b 7$ and b11 which will be traversed five times. Further we know the direction in which they are traversed; positive or negative. Given the groups will be linked, we wish to finish a group moving forward in a counter clockwise direction, equivalent to a positive $B$ edge. The edge points of $B$ can be divided into the following five groups according to their appearance in consecutive counter clockwise direction or clockwise direction on the slope diagram. 
1. b12, b1, b2 (counter clockwise)

2. b3, b4 (counter clockwise)

3. b5, b6, b7 (counter clockwise)

4. b8 (clockwise)

5. b9, b10, b11 (counter clockwise)

For each group we follow the precedence of $A$ searching for the next $B$ edge in the sequence. For example for group 1, we begin with the first $B$ edge on the list at the occurrence that follows a1. This ensures we end on a positive $B$ edge, i.e we have not broken the +,-,+ sequence of b12. The next $B$ edge is b1, hence we traverse b12, a2, a3, b12, a4, b12, a5, a6, b1. Note that the admissible $B$ edges that can be included are either b12 or b1, if we had encountered other $B$ edges on route to b1, they would have been ignored. This can be observed in other groups. Next, we search for b2, which is encountered directly after b1. Although, b12, b1 and b2 have been found, we know we must traverse each three times, hence the search continues through a7, -b2, a8, -b1, a9, a10, b1, a11, b2. In order to link each group, some additional $A$ edges need to be added. For group 1 the final $A$ edge is a12 and the first $A$ edge in group 2 is a7, hence we must return through $-\mathrm{a} 12$ to $-\mathrm{a} 7$ to retain the precedence order. The full edge list is detailed below, where the $A$ edge that precedes the starting $B$ edge is included in square brackets to indicate the starting point, but is not part of the edge list. The linking edges are underlined. 

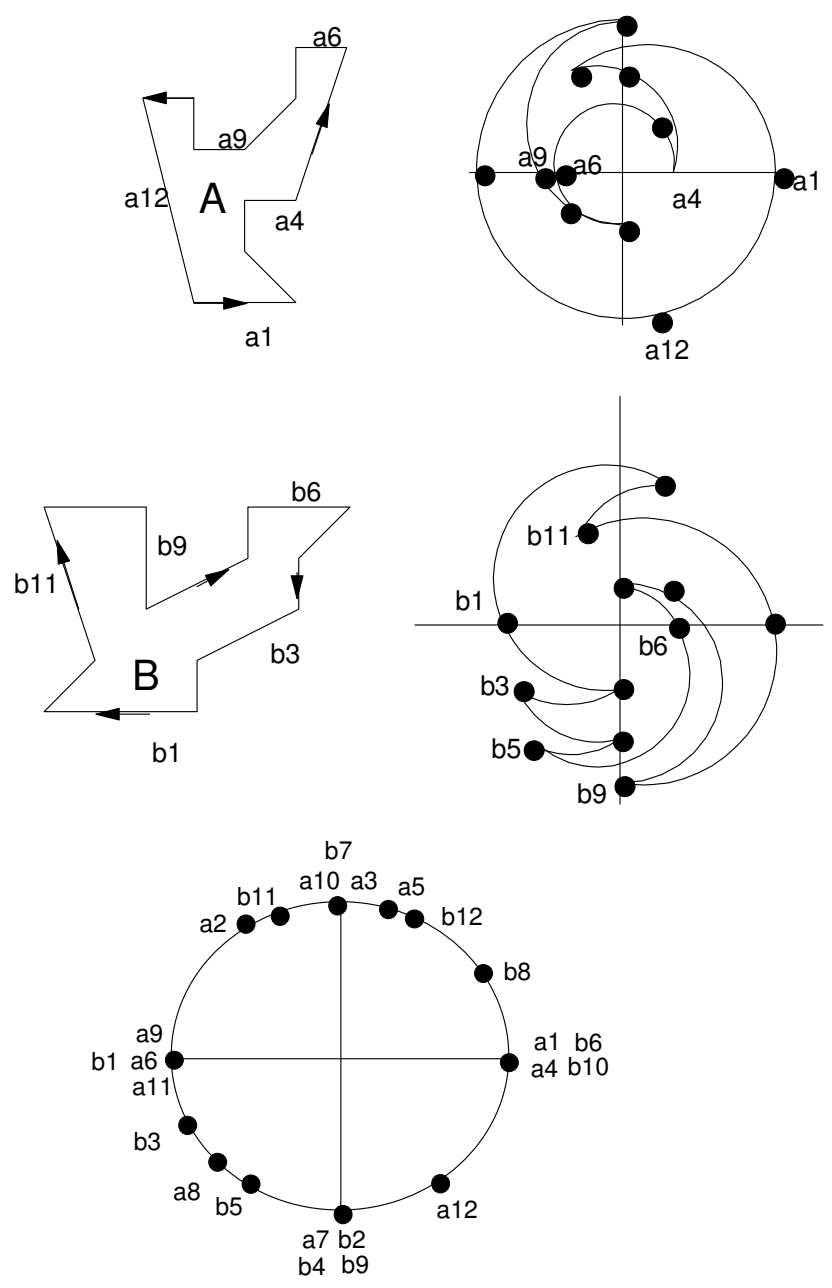

Figure 5: Complex case of two polygons with more than one concavity in each polygon

1. [a1], b12, a2, a3, -b12, a4, b12, a5, b1, a6, b2, a7, -b2, a8, -b1, a9, a10, b1, a11, b2, - a11, -a10, -a99, -a7

2. [a6], b3, b4, a7, -b4, a8, -b3, a9, a10, a11, b3, b4, -a11, -a10, -a9, -a7

3. [a6], b5, a7, -b5, a8, a9, a10, a11, b5, a12, b6, a1, b7, a2, -b7, a3, -b6, a4, b6, a5, b7, a6, a7, a8, a9, -b7, a10, b7, -a10,-a9, -a7, -a6, -a5

4. [-a5], b8, -a4, -b8, -a3, -a2, b8, a2, a3, a4, a5 
5. [a6], b9, a7, -b9, a8, a9, a10, a11, b9, a12, b10, a1, b11, a2, -b11, a3, -b10, a4, b10, a5, b11, a6, a7, a8, a9, -b11, a10, b11, -a10, -a9, -a7, -a6, -a5, -a4, -a3, -a2

The procedure to find the Minkowski sum of two polygons $A$ and $B$ is given below. Note that if a group of $B$ edges have continuous counter clockwise order, it is labeled as positive, otherwise it is labeled as negative. A positive group traverses the slope diagram of $A$ in counter clockwise order including positive $A$ edges. A negative group follows the slope diagram in clockwise order including negative $A$ edges. Only the positive procedure is included here. For efficiency, the procedure in $\operatorname{Mink}(\mathrm{Q}, \mathrm{R}$,positive) traverses the slope order list following the precedence of $A$ recording the $B$ edges encountered along the way to provide list, $s$. This process allows us to know the number of times each $B$ edge is encountered and in what direction. Further list $s$ can be used to create the correct sequence of $A$ and $B$ edges without searching the slope order a second time.

\section{Algorithm 1: Algorithm to generate the Minkowski Sum}

Step 1: Replace B by $-\mathrm{B}$, i.e. replace all co-ordinates $\left(x_{B}, y_{B}\right)$ of $B$ by $\left(-x_{B},-y_{B}\right)$.

Step 2: Starting at the lowest point on each polygon. Label the edges in counter clockwise order.

Calculate the angle $\theta(i)$ of each edge, $i$, from the horizontal in a counter clockwise direction.

For each edge $i$, let $\alpha(i)=\theta(i)-\theta(i-1)$.

If $\alpha(i)>180$ then $\alpha(i)=\alpha(i)-360$.

If $\operatorname{sign}(\alpha(i)) \neq \operatorname{sign}(\alpha(i-1))$ mark $i$ as a turning point. 
If any turning points have been detected then polygon is non-convex.

Sort the edges into angle order to form sort_list $(P)$, where $P=A$ or $-B$.

Step 3: Let $\left(b_{k_{1}+1}, \cdots, b_{k_{n}+1}\right)$ be the set of turning points on the slope diagram of polygon $B$. Then polygon $B$ can be divided into groups $B_{l}=\left(b_{k_{1}+1}, b_{k_{1}+2}, \cdots, b_{k_{2}}\right)$, $B_{2}=\left(b_{k_{2}+1}, b_{k_{2}+2}, \cdots, b_{k_{3}}\right), \ldots, \quad B_{n}=\left(b_{k_{n}+1}, b_{k_{n}+2}, \cdots, b_{k_{1}}\right) \quad$ according to them being consecutive counterclockwise direction or consecutive clockwise direction.

Step 4: For each group $B_{j},(j=1, \ldots, n)$, call $\operatorname{Mink}\left(A, B_{j}\right.$, positive $)$ or $\operatorname{Mink}\left(A, B_{j}\right.$, negative $)$ according to group $B_{j}$ being counter clockwise or clockwise respectively. We obtain $\mathrm{Seq}_{j}$.

Step 6: Link Seq_list $\left(\mathrm{A}, \mathrm{B}_{1}\right), \ldots$, Seq_list $\left(\mathrm{A}, \mathrm{B}_{\mathrm{n}}\right)$ with additional $A$ edges one by one.

If Seq_list $\left(\mathrm{A}, \mathrm{B}_{\mathrm{j}}\right)$ is positive, insert negative $A$ points to link $S e q_{i}$ with $S e q_{i+1}$. else Seq_list $\left(\mathrm{A}, \mathrm{B}_{\mathrm{j}}\right)$ is negative, insert positive $A$ points to link $S e q_{i}$ with $S e q_{i+1}$.

$\operatorname{Mink}(Q, R$, positive).

Step 1 : merge sort_list(Q) and sort_list(R) to form merge_list(Q,R)

Step 2: set $i=1, \mathrm{k}=1$, direction $=1, \mathrm{~s}_{1}=\mathrm{q}_{1}$

Step 3: Set $i=i+1$

Search merge_list $(\mathrm{Q}, \mathrm{R})$ for $\mathrm{q}_{\mathrm{i}}$ moving forward if direction $=1$ and backwards if direction $=-1$

if R edge, $r_{j}$, set $k=k+1, s_{k}=$ direction $\times r_{j}$

When $q_{i}$ is encountered, if $i=1$, go to step 4

Otherwise set $k=k+1, s_{k}=q_{i}$ 
If $q_{i}$ is a turning point in $\mathrm{Q}$, set direction $=-$ direction

Repeat step 3

Step 4: Let starting edge $r_{1}$ be in position $s_{i}$ in sequence

Set $j=1$, next $=2$, direction $=1, \operatorname{seq}_{1}=s_{i}$

Step 5: Set $i=i+1$, if $i>k$, set $i=1$

If $s_{i}$ is from Q, $j=j+1, \operatorname{seq}_{j}=s_{i}$

If $s_{i}$ is a turning point in $\mathrm{Q}$, direction $=-$ direction, next $=$ next + direction

If $s_{i}=$ direction. $\mathrm{r}_{\text {next }}, j=j+1$, se $q_{j}=s_{i}$, next $=$ next + direction

If all $s_{i}$ edges have been allocated to $s e q_{j}$, return $s e q_{1}$ to $s e q_{j}$ as Seq_list(Q,R)

Otherwise, repeat step 5

\section{COMPUTING THE BOUNDARY OF THE NFP}

The resulting Minkowski sum is a complex (self crossing) polygon where the edges include all the edges of the nofit polygon and some internal points. In this section we describe a new method for identifying the true edges of the NFP.

Figure $6 \mathrm{~b}$ illustrates the outcome of the Minkowski sum procedure described in the previous section, for two simple polygons drawn in 6a. Clearly there are many edges to untangle. However, using some of the properties we know about the NFP and the boundary addition method we can quickly remove many of these edges.

Property 1. As detailed earlier in the paper, the NFP can be described as the path of a reference point on $B$ as it slides around the edges of $A$. Further, the sliding motion is always in the same direction (i.e. counter clockwise) and as a result the path mapped by the reference point on $B$ is also in one direction. 
Property 2. The slope diagram representation, used in boundary addition, indicates that if edge $b_{j}$ appears between edges $\left(a_{i}, a_{i+1}\right)$, this corresponds to the physical condition that edge $b_{j}$ slides along vertex $\left(a_{i}, a_{i+1}\right)$. When the direction from $a_{i}$ to $a_{i+1}$ is counter clockwise and edge $b_{j}$ is positive, the corresponding sliding condition is that the convex vertex $\left(a_{i}, a_{i+1}\right)$ slides along $b_{j}$. Otherwise, the negative edge $b_{j}$ corresponds to the condition that the concave vertex $\left(a_{i}, a_{i+1}\right)$ slides along edge $b_{j}$. Clearly an edge cannot slide along a concave vertex without creating overlap between the polygons.

It can be deduced from these two properties that any negative edges cannot be part of the boundary of the NFP and can be removed. Further, the linking edges between sequences can also be removed since their inclusion is to define the correct starting position of the next sequence and they do not represent potential sliding between the polygons. Figure $6 \mathrm{c}$ illustrates the Minkowski edge list with the redundant edges removed. With this understanding, we can develop intuitively a new method to identify the boundary of the NFP by only considering useful parts of the derived Minkowski sum edge list. 


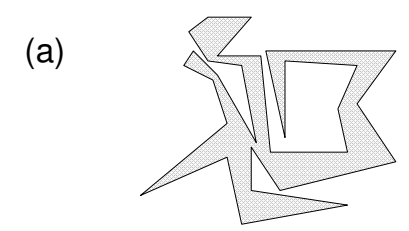

Polygon A

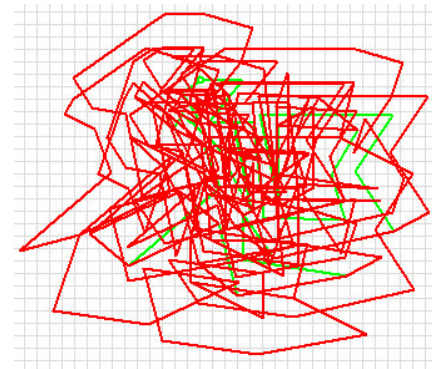

(b) Minkowski edge list

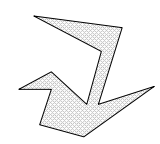

Polygon B

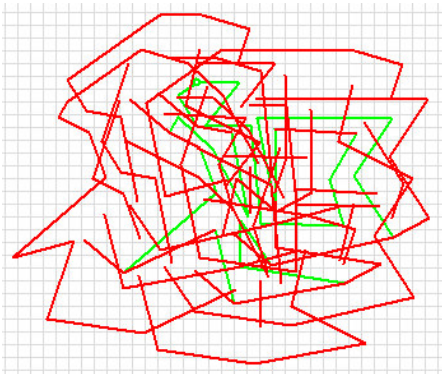

(c) Minkowski sum after removal of redundant edges

(d)

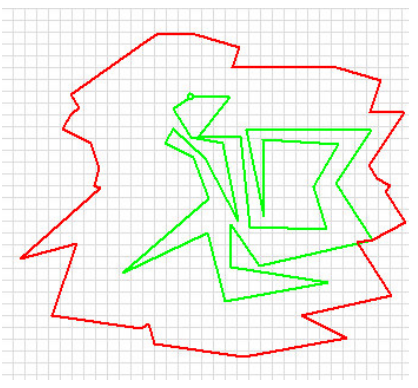

Figure 6: A large example of the Minkowski sum edge list and track line traces

In order to introduce this method, we recall briefly some terms introduced by Ramkumar (1996). A state is a pair consisting of a position $s$ in the plane and a direction s. A move is a set of states with constant direction and position varying along a line segment parallel to the direction. A turn is a set of states with constant position and direction varying along an arc of the circle of directions. A polygonal trip consists of a continuous sequence of moves and turns; the trip is closed if it starts and ends at the same state. A polygonal tracing is a collection of closed polygonal trips. We think of each loop 
of a tracing as being traversed by a car which always faces in the direction of the state it is currently following.

The first step is to break up the Minkowski sum into polygonal trips according to the continuous sequence of moves and turns. Those that cannot be part of the boundary of the NFP, according to the properties described above, are discarded. This is equivalent to removing the negative and additional $A$ edges.

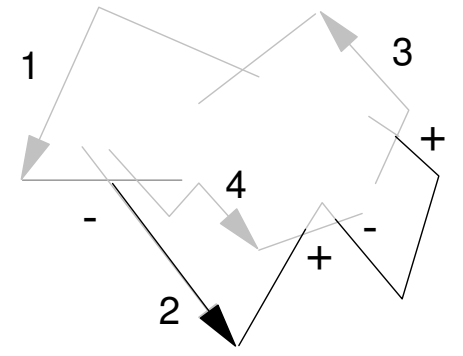

(a) Identify each intersection as entering or leaving the trip

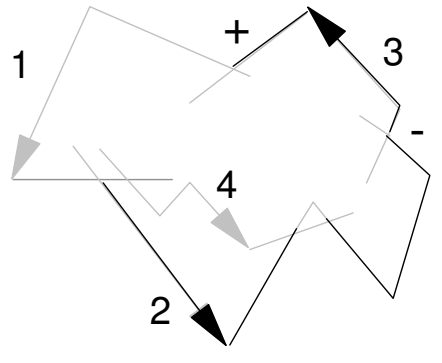

(b) Truncate each trip retaining parts between '-' and ' + '

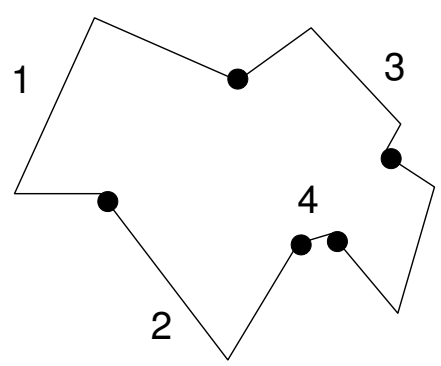

(c) Repeat and link parts

Figure 7: Procedure for removing internal edges from the Minkowsi sum

The second step of the procedure requires the identification of all the intersection points between the polygonal trips. Each intersecting point is marked with a '-', indicating it is entering the trip, or with a '+', indicating it is leaving the trip. Consider the example in figure $7 \mathrm{a}$, where the current trip is trip 2. Imagine standing at the beginning of the first edge of trip 2 facing along the edge, then we can consider that trip 1 intersects trip 2 from right to left. Trip 1 is said to enter trip 2 and is marked with '-'. Continuing along trip 2 we eventually meet an intersection with trip 4, where trip 4 intersects from left to right. Trip 4 is leaving trip 2 and marked with '+'. Hence, entering 
a trip means the beginning part of the intersecting edge is on the right side of the trip edge and leaving corresponds to the beginning part of the intersecting edge is on the left side of the trip edge. The fragments of trips that span a '-' intersection to a ' + ' intersection are kept to form the boundary of the NFP. All other fragments are discarded.

Finally, fragments that share end points are linked. Only those parts, which form a cycle, can be taken as the boundary of the NFP. In the case where cycles represent holes in the NFP, we implement a simple direct test of whether the two component polygons overlap when the reference point of B is located on a vertex of the hole.

The algorithm for finding the boundary of the nofit polygon can be summarized in Algorithm 2 and 3 as below:

Algorithm 2: Algorithm for breaking Minkowski sums into track line trips:

Step 1: Let $i=0$ be the index number of Minkowski sums obtained. Let $j=0$ be the number of track line trips, $n_{j}$ be the total edges in track line trip $j$ and $k=0$ be the index of each track line trip.

Step 2: Search forward in Minkowski Sum for positive $s_{i}$, which corresponds to a track line. If $s_{i}$ can be found, set $t_{j_{k}}=s_{i}$, else go to Step 4 .

Step 3: Set $i=i+1$ and $k=k+1$. If $s_{i}$ is positive and corresponds to a track line, repeat step 3, else set $n_{j}=k, j=j+1$ and $k=0$, repeat Step 2 .

Step 4: Return $t_{j_{0}}$ to $t_{j_{n_{j}}}$ as track line trip $T_{j}$. 
The computational complexity of Algorithm 2 is $O(N)$, where $N$ is the number of edges of Minkowski sums.

Algorithm 3: Algorithm for finding the boundary of the NFP from track line trips:

Step 1: Let trip $T_{j}$, contain $\operatorname{seg}_{j n_{j}}$ segments.

For all $i$ and $j$ where $i \neq j$,

If $s e g_{j k_{j}}$ intersects $s e g_{i k_{i}}$, let $p_{j r_{j}}$ be the intersection points on $T_{j}$

If $p_{j r_{j}}$ crosses right to left set $\operatorname{sign} n_{j r_{j}}=-1$, else set $s i g n_{j r_{j}}=+1$.

Step 2: For each $T_{j}$,

If $\operatorname{sign}_{j r_{j}}=-1$ and $\operatorname{sign}_{j r_{j}+1}=+1$. Store all segments between the intersection points, $\left\{p_{j r_{j}-1}, s_{j k_{j}}, \cdots, s_{j k_{j}+m}, p_{j r_{j}}\right\}$, into $\operatorname{frag}_{i}=\left\{f_{i 1}, \ldots, f_{i k}, \cdots, f_{i l_{i}}\right\}$, where $l_{i}$ is the number of segments in frag $_{i}$.

Step 3: For all frag $_{i} \neq 0$, we

if $f_{i 1}=f_{j l_{j}}$, frag $_{i}=\operatorname{frag}_{j}+\operatorname{frag}_{i}=\left\{f_{j 1}, \cdots, f_{j l_{j}} \cdots, f_{i l_{i}}\right\}$, frag $_{j}=0$.

if $f_{j 1}=f_{i l_{i}}, \operatorname{frag}_{i}=\operatorname{frag}_{i}+\operatorname{frag}_{j}=\left\{f_{i 1}, \cdots, f_{i l_{i}} \cdots, f_{j l_{j}}\right\}$, frag $_{j}=0$.

if $f_{i 1}=f_{j l_{j}}$ and $f_{j 1}=f_{i l_{i}}$ then form cycle $_{k}$ and set frag $_{i}=$ frag $_{j}=0$

Repeat step 3 until all frag $_{i}=0$.

Step 4: For each cycle ${ }_{k}$,

Locate reference point of polygon $B$ on one of the vertexes of cycle $_{k}$.

Discard the cycle $_{k}$ if the two polygons overlap 
It is important to note that the above algorithm is able to identify the outer face of the Minkowski sums, holes inside the outer face, a single point that represent an exact fit, and exact slides represented as a single line. The latter two are often referred to as degenerate cases.

\section{Degenerate cases}

The degenerate cases, in general, refer to combinations of polygons that can fit together like a jigsaw, resulting in a single point of fit within the NFP, or where one piece can slide into or within a concavity in one direction only, resulting in a line either extending from the edge of the NFP or within.

(a)

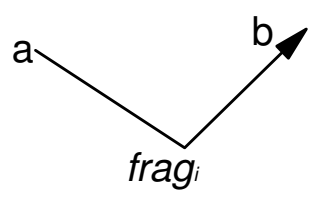

(b)

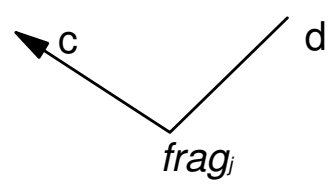

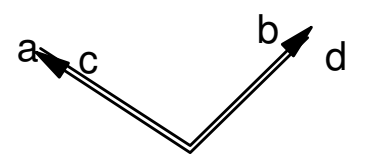

(c)

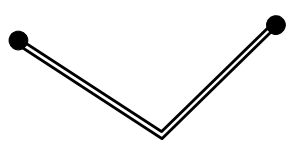

Figure 8: Coinciding fragments to indicate exact slide in NFP

An exact slide can be identified when two fragments, obtained from Algorithm 3, step 2, coincide. Figure 8a illustrate frag $_{i}$ and frag $_{j}$ with start points, a and d, and end points, $b$ and $\mathrm{c}$ respectively. If the start and end points from each fragment coincide, as shown in figure $8 \mathrm{~b}$ they can be linked into a cycle (figure $8 \mathrm{c}$ ) in step 3 . The cycle is validated as part of the boundary of the NFP in step 4 . 
In the case of a single point, further calculation is required in Algorithm 3, step 1. When all the intersection points between segments of the trip are calculated, we also identify special intersection cases as shown in figure 9 , where trips $T_{i}$ and $T_{j}$ intersect each other at point A. It is necessary to test all intersection points of this type to identify if it is an exact fit. In our experience, intersection points such as A seldom occurred within the intersection condition.

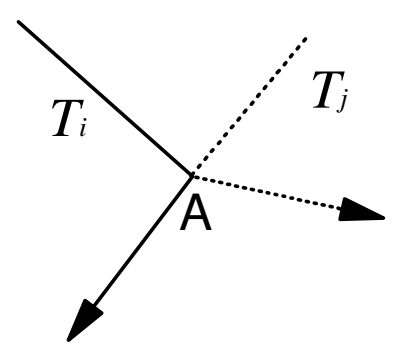

Figure 9: Edge intersection to indicate exact nesting point in NFP

\section{THE INNER FIT POLYGON}

The inner fit polygon represents the feasible placement positions of one polygon, $B$, inside another polygon, $A$. An example of this is given in figure 10. This is useful for obstacle recognition in robot motion planning and if pieces are being packed inside an irregular shape, for example, shoe manufacturing from leather hides. 


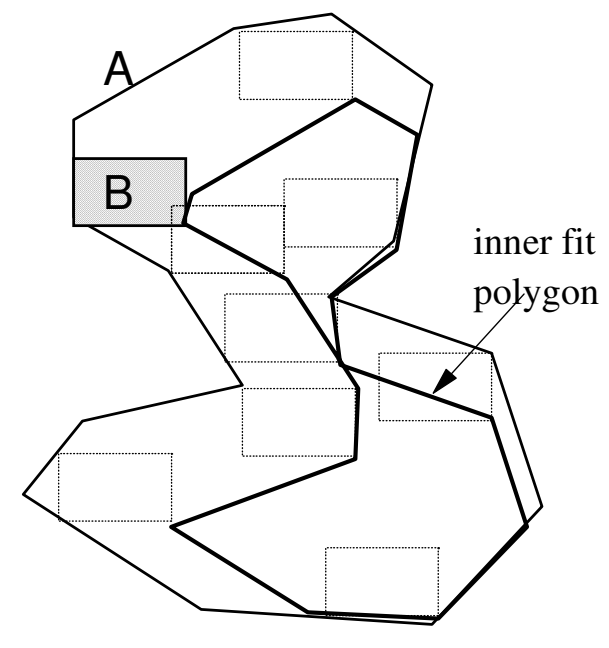

Figure 10: An inner fit polygon

The described algorithm can be used to calculate the inner-fit polygon with the following minor amendments.

(i) Reverse the orientation of polygon $A$ so that it has clockwise direction.

(ii) The algorithm should have the positive direction for $A$ as clockwise. However, clockwise is still the negative direction for $B$

The rationale for these changes can be demonstrated in figure 11. The inner-fit polygon is equivalent to $B$ sliding inside a concavity of $A$. As illustrate in figure 11 , the concavity has clockwise orientation. Further a concavity translates to a clockwise turn in the slope diagram where the edges of the concavity remain positive, while any edges from the other polygon encountered during that turn are negative. 


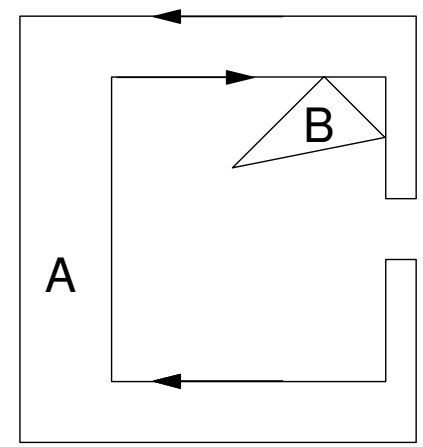

Figure 11: the equivalence between the inner-fit polygon and the NFP inside a concavity

\section{EMPICICAL ANALYSIS}

In order to evaluate the effectiveness of our new approach, we generated all the nofit polygons for the benchmark data sets found on the ESICUP (2005) website. All were generated correctly and the computation times for every combination of each data set are provided in table 1. The procedure was coded in Visual Studio $\mathrm{C}++$ and the instances were run on a pc with $512 \mathrm{MB}, 1.6 \mathrm{GHz}$.

\begin{tabular}{|c|c|c|c|c|}
\hline No. & CASE & No. of piece types & Ave no. of edges & TIME (s) \\
\hline 1 & Albino_hopper & 8 & 7.25 & 0.07 \\
\hline 2 & Blaz_topos & 7 & 6.28 & 0.04 \\
\hline 3 & Dagli_hopper & 10 & 6.3 & 0.07 \\
\hline 4 & Dighe_hopper & 10 & 4.7 & 0.04 \\
\hline 5 & Dighe_hopper-1 & 16 & 3.8 & 0.07 \\
\hline 6 & Fu_hopper & 12 & 3.6 & 0.04 \\
\hline 7 & Han_hopper & 20 & 6.95 & 0.33 \\
\hline 8 & Jakobs_hopper & 25 & 5.8 & 0.42 \\
\hline
\end{tabular}




\begin{tabular}{|c|c|c|c|c|}
\hline 9 & Mao_hopper & 9 & 9.2 & 0.15 \\
\hline 10 & Marques_hopper & 8 & 7.1 & 0.07 \\
\hline 11 & Poly_hopper & 75 & 4.8 & 2.00 \\
\hline 12 & Shapes_topos & 4 & 8.7 & 0.03 \\
\hline 13 & Shirts_topos & 8 & 6.6 & 0.06 \\
\hline 14 & Swim_topos & 10 & 22.8 & 0.93 \\
\hline 15 & Trousers_topos & 17 & 5.1 & 0.10 \\
\hline
\end{tabular}

Table 1: generation times of all NFPs in benchmark data sets

In addition we have extensively tested our approach on new instances designed to involve characteristics such as, a large number of edges, interlocking positions, exact sliding, jigsaw type fits, and concavities the turned more than 360 degrees. All NFPs were successfully generating, none taking more than once second to generate. The results can be found in the figures $10-13$.

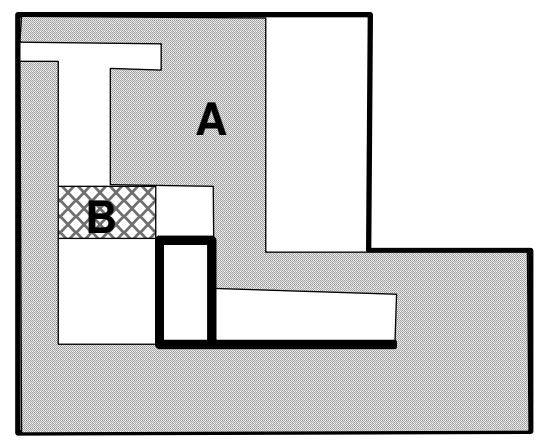

(a)

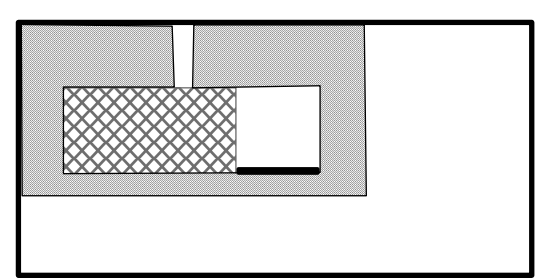

(b)

Figure 10: a) The nofit polygon contains a hole and an exact slide along the bottom edge of the concavity. b) The nofit polygon contains an exact slide. 


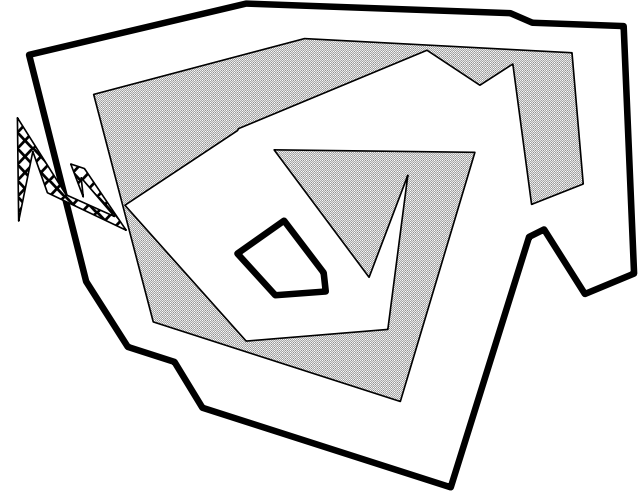

(a)

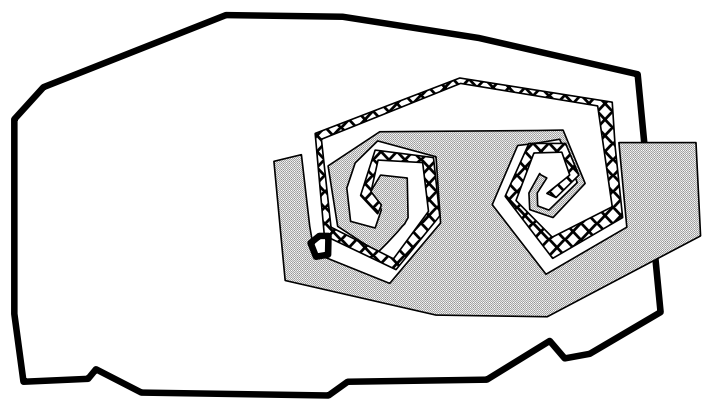

(b)

Figure 11: a) The nofit polygon contains a hole. The concavity in polygon A turns through more than $360^{\circ}$. b) Nofit polygon contains a hole. Both A and B have concavities that turn greater than $360^{\circ}$ and interlock with each other.

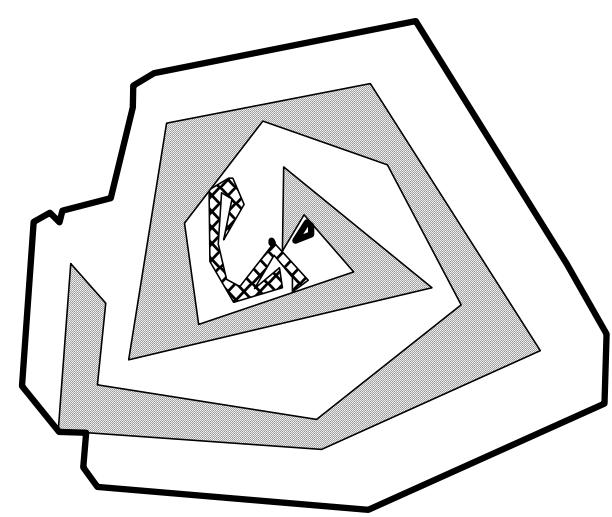

(a)

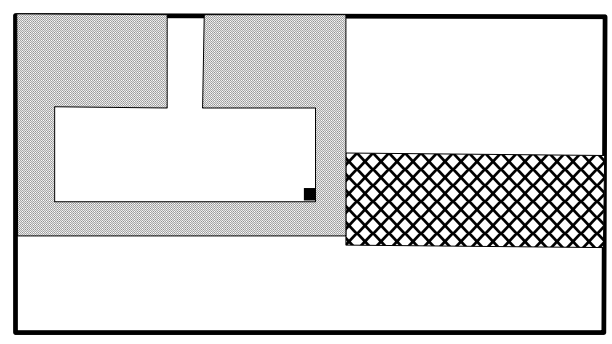

(b)

Figure 10: a) The nofit polygon contains a hole and an exact fit indicated by the illustrated position of polygon B. b) Polygon B fits exactly in the concavity of polygon A. 


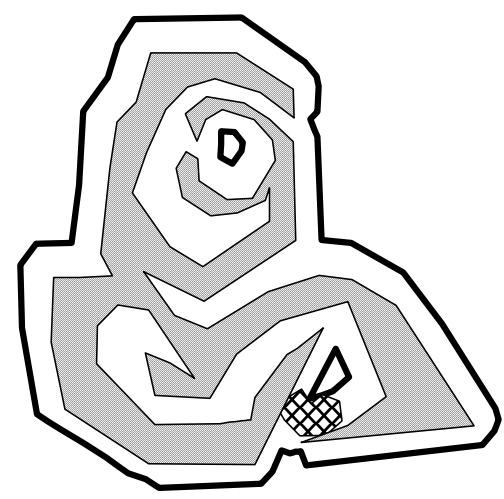

(a)

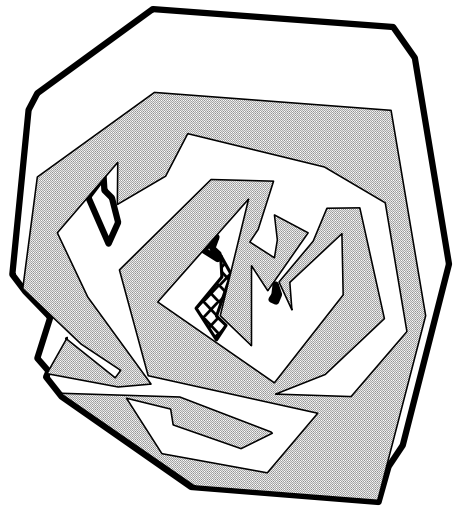

(b)

Figure 11: Both nofit polygons contain multiple holes. Polygon A has concavities within concavities.

\section{CONCLUSIONS}

In this paper we have described a new approach of finding the nofit polygon. Empirical analysis demonstrates that computational times are realistic and that the approach is robust in dealing with known degenerate cases and new difficult cases such as spiraling concavities. The method is theoretically underpinned by the concept of Minkowski sums and builds on the work of Ghosh by adapting his boundary addition theorem into an algorithm procedure. It improves on the work of Bennell, Dowsland and Dowsland by finding the Minkowski sum in a single procedure and removing any ambiguity over which edges should be included in the repair procedure. Finally the paper provides a new, simple and robust procedure for the removal of internal edges and 
identification of holes. All approaches are described in detail, illustrated by example and the summary code is provided.

\section{REFERENCES}

Adamowicz M, Albano A., 1976, Nesting two dimensional shapes in rectangular modules, Computer Aided Design, 8(1), 27-33.

Agarwal, P.K., Flato, E., Halperin, D., 2002, Polygon decomposition for efficient construction of Minkowski sums, Computational Geometry Theory and Applications, 21, 39-61

Babu, R.A., Babu, R.N., 2001, A genetic approach for nesting of 2-D parts in 2-D sheets using genetic and heuristic algorithms, Computer-Aided Design, 33, 879-891

Bennell, J.A., Dowsland, K.A., Dowsland, W.B., 2001, The irregular cutting-stock problem - a new procedure for deriving the nofit polygon, Computers and OR, 28, 271287.

Bennell, J.A., 1998, Incorporating problem specific knowledge into a local search framework for the irregular shape packing problem, Ph.D. dissertation, EBMS, University of Wales, Swansea, UK 
Burke, E.K., Hellier, R.S.R, Kendall, G. and Whitwell, G., 2005, Complete and robust no-fit polygon generation for the irregular stock cutting problem, working paper, ASAP, School of Computer Science, University of Nottingham, UK

Cunninghame-Green, R., 1989, Geometry, Shoemaking and the milk tray problem, New Scientist, $12^{\text {th }}$ August, no. $1677,50-53$.

ESICUP, 2005, European working group on cutting and packing. http:/www.apdio.pt/sicup.

Ghosh, P.K., 1993, A unified computational framework for Minkowski operations. Computers and Graphics, 17(4), 357-78.

Ghosh, P.K., 1991, An algebra of polygons through the notion of negative shapes. CVGIP: Image Understanding, 54(1), 119-44.

Konopasek M. (1981), Mathematical Treatments of Some Apparel Marking and Cutting Problems, U.S. Department of Commerce Report 99-26-90857-10.

Li, Z., Milenkovic, V.J., Daniels, K., 1995, Compaction and separation algorithms for non-convex polygons and their applications, European Journal of Operational Research, $84,539-561$. 
Mehadevan, A., 1984, Optimization in computer aided pattern packing, Ph.D. dissertation, North Carolina State University.

Oliveira J. F., and Ferreira J. S., 1993, Algorithms for nesting problems, Applied Simulated Annealing, R.V.V. Vidal (ed), Lecture Notes in Econ. and Maths Systems 396, Springer Verlag, 255-274.

Ramkumar, G.D., 1996, An algorithm to compute the Minkowski sum outer face of two simple polygons, Proceedings of the $12^{\text {th }}$ Annual Symposium on Computational Geometry, 234-241.

Stoyan, Y., Terno, J., Scheithauer, G., Gil, N., Romanova, T., 2001, Phie functions for primary 2D-objects, Studia Informatica Universalis, 2, 1, 1-32

Stoyan, Y., Scheithauer, G., Gil, N., Ramanova, T., 2002, Phi-functions for complex 2Dobjects, Technical Report, MATH-NM-2-2002, April 2002, Technische Universitat Dresden.

Wäscher,G. Haußner H., Schumann, H., 2005, An improved typology of cutting and packing problems, working paper, Faculty of Economics and Management, Otto von Guericke University, Magdeburg. 
Watson P.D. and Tobias, A.M., 1999, An efficient algorithm for the regular W1 packing of polygons in the infinite plane, Journal of the Operational Research Society, 50. 10541062.

\section{Acknowledgements}

The authors would like to acknowledge the financial support of the Engineering and Physical Sciences Research Council. 